\title{
Patrón de cicatrización neointimal de un stent farmacoactivo en el hematoma coronario. ¿Cómo lo haría?
}

\author{
Neointimal healing pattern of a drug-eluting stent in a coronary \\ hematoma. How would I approach it?
}

\author{
Santiago Jiménez Valero* \\ Servicio de Cardiología, Hospital Universitario La Paz, Madrid, España
}

VÉASE CONTENIDO RELACIONADO:

https://doi.org/10.24875/RECIC.M19000023

https://doi.org/10.24875/RECIC.M19000025

\section{¿CÓMO LO HARÍA?}

El caso que se presenta resulta muy ilustrativo sobre una complicación a la que, afortunadamente, en la actualidad nos enfrentamos en raras ocasiones: la oclusión aguda del vaso tras el intervencionismo coronario. En este contexto, la utilización de técnicas de imagen intracoronaria resulta fundamental para determinar los mecanismos de dicha oclusión y guiar el tratamiento. Probablemente los mecanismos más conocidos sean los relacionados con la trombosis del stent, como la infraexpansión o la disección en los bordes, pero en este caso se describe una causa menos frecuente: la progresión distal de un hematoma intramural coronario.

Podría debatirse si se trata de un hematoma intramural iatrogénico, relacionado con el daño producido por el stent en la pared coronaria, la potente antiagregación utilizada, etc., o bien si un hematoma espontáneo podría subyacer en la lesión "culpable» de la coronaria derecha. Aunque la imagen angiográfica no es la típica de disección/hematoma intramural espontáneo, hay algunos casos indistinguibles de lesiones coronarias ateroscleróticas (figura 1), que se encuadran en el tipo III de la clasificación de Saw et al. ${ }^{1}$ y que requieren la utilización de técnicas de imagen intracoronaria para establecer el diagnóstico. La utilización previa al intervencionismo de técnicas de imagen en lesiones causantes de síndrome coronario agudo puede aportar información importante para guiar el procedimiento e incluso cambiar la actitud terapéutica. Por ejemplo, en este caso, y aunque este aspecto pueda ser discutible, si se demostrase que la causa es un hematoma espontáneo, teniendo en cuenta la estabilidad clínica de la paciente, sin dolor ni elevación del ST y con flujo coronario normal, podría plantearse un tratamiento conservador, sin implante de stent, para permitir la potencial «curación» espontánea del vaso.

En cuanto a la estrategia en el caso, coincido con los autores en la utilización de la ecocardiografía intracoronaria (IVUS) como técnica de imagen, puesto que distalmente al stent el vaso presenta una oclusión total, lo que dificulta la realización de una tomografía de coherencia óptica (OCT). Además de confirmar el diagnóstico de hematoma intramural, la IVUS permitiría evaluar su extensión distal, el compromiso de ramas laterales $y$, de cara a la necesidad de intervencionismo, confirmar la posición de la guía en la verdadera luz.

En este caso se optó por un tratamiento con stent directo, que probablemente sea la opción más segura para recuperar el flujo distal y prevenir la reoclusión del vaso. Sin embargo, algunos autores recomiendan implantar un stent solo como alternativa cuando no puede recuperarse el flujo mediante dilatación con balón. Las razones para ello son varias; en primer lugar, es frecuente que el implante del stent provoque la progresión distal o proximal del hematoma, o disección, que puede precisar múltiples stents, producir la oclusión de ramas laterales y a veces ocasionar situaciones de alto riesgo, como la progresión hasta el tronco izquierdo. Además, si se consigue recuperar un flujo distal adecuado mediante dilatación con balón, evitando así la isquemia miocárdica, se ha descrito en numerosos casos que puede producirse la "curación» del vaso, con resolución completa del hematoma, evitando así las posibles complicaciones a largo plazo relacionadas con la implantación de stents (reestenosis, trombosis, etc.). Otra opción que se ha planteado para recuperar el flujo distal es la «fenestración» del hematoma mediante un balón de corte o un balón scoring ${ }^{2}$. La fenestración permite la descompresión del hematoma y con ello mejora el flujo en la luz verdadera, que se encuentra comprimida, y puede reducirse el riesgo de progresión del hematoma.

En respuesta a la pregunta «¿cómo lo haría?», mi estrategia en este caso sería la siguiente: en el primer procedimiento no habría utilizado un inhibidor IIb/IIIa; solo lo habría empleado en caso de intervencionismo complejo o alta carga de trombo. Hay poca experiencia sobre su seguridad en combinación con ticagrelor o prasugrel, y las guías de práctica clínica solo lo recomiendan con clase IIb, nivel de evidencia $\mathrm{C}$, en pacientes con síndrome coronario agudo tratados con intervencionismo percutáneo y que no hayan recibido un inhibidor $\mathrm{P}_{2} \mathrm{Y}_{12}$ como segundo antiagregante. En el segundo procedimiento realizaría IVUS para evaluar el mecanismo de la oclusión del vaso y,

\footnotetext{
* Autor para correspondencia: Servicio de Cardiología, Hospital Universitario La Paz, Paseo de la Castellana 261, 28046 Madrid, España. Correo electrónico: sjvcardio@yahoo.es (S. Jiménez Valero). 


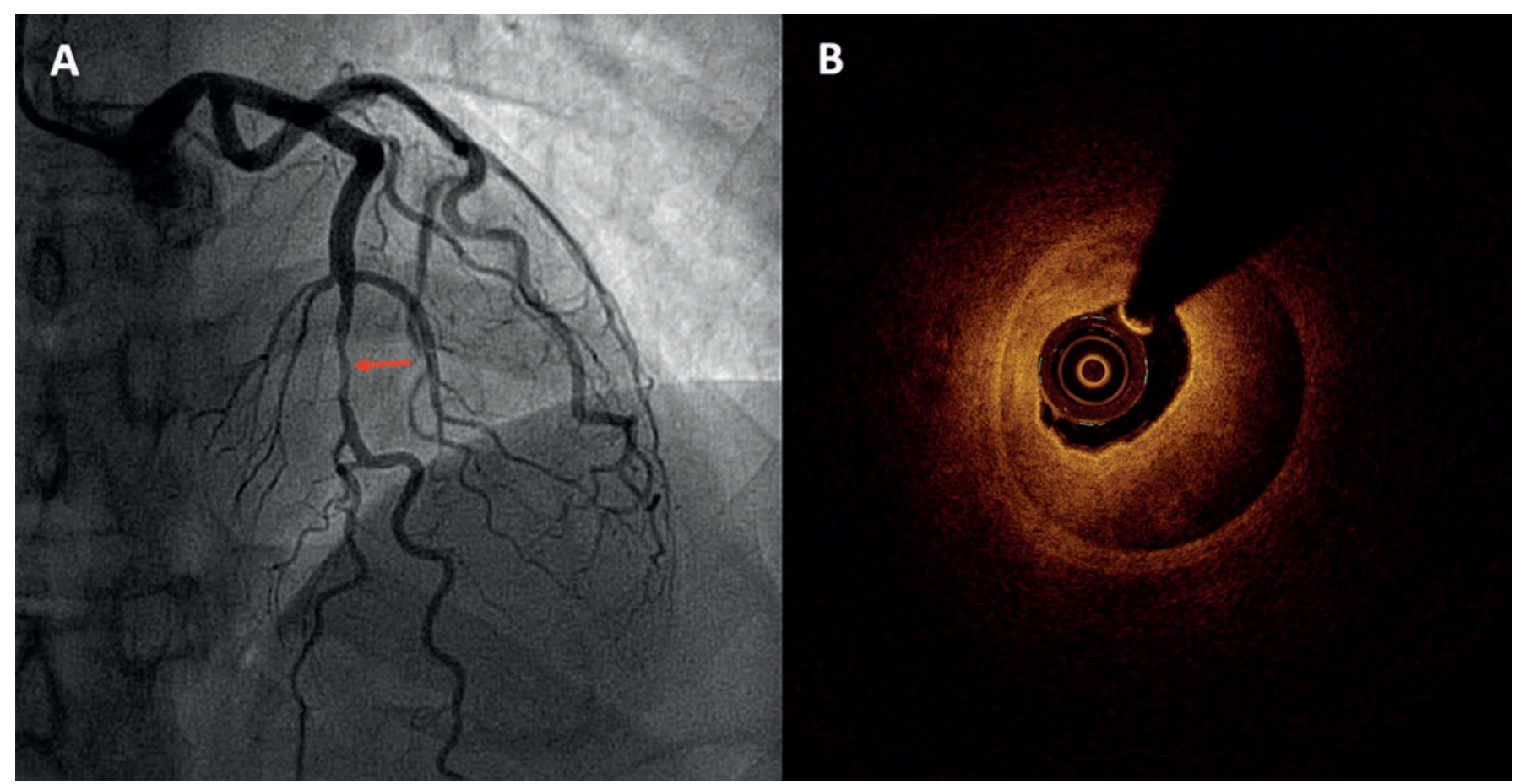

Figura 1. A: coronariografía en un paciente con síndrome coronario agudo sin elevación del ST que muestra una lesión grave en la descendente anterior media (flecha). B: la tomografía de coherencia óptica evidenció un hematoma intramural como causa de dicha lesión.

al diagnosticar el hematoma intramural, determinar su extensión distal. Si distalmente se identificase con IVUS una zona sana, implantaría un stent de diámetro ajustado al tamaño del vaso distal en la zona sana, liberado a presión nominal, hasta solaparse con el stent previo, con el objetivo de «contener» el hematoma y evitar la progresión distal. En el caso de precisar más de un stent, implantaría primero el más distal, asegurando «aterrizar» en una zona sana. Si por el contrario la IVUS mostrase una afectación difusa hasta las ramas distales de pequeño calibre, para recuperar el flujo distal intentaría en primer lugar una dilatación con balón. En este caso se describe daño hasta la bifurcación descendente posterior/posterolateral, por lo que intentaría avanzar guías a ambas ramas y habría optado por dilatación con balón. Si no se recuperara el flujo, intentaría la fenestración con un balón de corte ligeramente infradimensionado. Si mediante estas medidas se consiguiese un flujo coronario normal, no implantaría el stent. Si no se recuperara el flujo distal, implantaría stents siguiendo la estrategia empleada por los autores en el caso, con stents largos e infradimensionados, intentando no ocasionar un mayor daño a la pared vascular.

Posteriormente programaría una nueva coronariografía, entre 1 y 3 meses después, para evaluar mediante OCT la presencia de hematoma y la aposición de los stents. Ya que se han utilizado intencionadamente stents infradimensionados, es previsible encontrar falta de aposición, pero incluso consiguiendo una aposición completa tras la implantación, con la reabsorción del hematoma puede desarrollarse una malaposición. Además, la OCT aportaría información sobre el recubrimiento neointimal precoz, que en muchas ocasiones puede incluso cubrir los struts no apuestos y solucionar espontáneamente la falta de aposición. En caso de malaposición grave, sobre todo si estuviese asociada a una falta de recubrimiento de los struts, consideraría la posdilatación del stent ${ }^{3}$ para facilitar el recubrimiento neointimal y potencialmente reducir el riesgo de trombosis.

\section{BIBLIOGRAFÍA}

1. Saw J, Mancini GBJ, Humphries KH. Contemporary Review on Spontaneous Coronary Artery Dissection. J Am Coll Cardiol. 2016;68:297-312.

2. Motreff $\mathrm{P}$, Ronchard T, Sanguineti F, et al. Coronary Artery Fenestration: A Promising Technique for Rescue Management of Spontaneous Intramural Hematoma With Luminal Compression. JACC Cardiovasc Interv. 2018;11:1905-1907.

3. Sanchez-Recalde A, Moreno R, Jimenez-Valero S. Stenting of spontaneous intramural coronary haematoma: long-term consequences. Eur Heart J. 2008;29:1593. 